\title{
Stacking order dependent electric field tuning of the band gap in graphene multilayers
}

\author{
A. A. Avetisyan, ${ }^{1,2, *}$ B. Partoens, ${ }^{1, \dagger}$ and F. M. Peeters ${ }^{1,3, \neq}$ \\ ${ }^{1}$ Departement Fysica, Universiteit Antwerpen, Groenenborgerlaan 171, B-2020 Antwerpen, Belgium \\ ${ }^{2}$ Department of Physics, Yerevan State University, 1 A. Manoogian, 0025 Yerevan, Armenia \\ ${ }^{3}$ Departamento de Física, Universidade Federal do Ceará, Caixa Postal 6030, Campus do Pici, 60455-900 Fortaleza, CE, Brazil
}

(Received 17 December 2009; published 18 March 2010)

\begin{abstract}
The effect of different stacking order of graphene multilayers on the electric field induced band gap is investigated. We considered a positively charged top and a negatively charged back gate in order to independently tune the band gap and the Fermi energy of three and four layer graphene systems. A tight-binding approach within a self-consistent Hartree approximation is used to calculate the induced charges on the different graphene layers. We found that the gap for trilayer graphene with the $A B C$ stacking is much larger than the corresponding gap for the $A B A$ trilayer. Also we predict that for four layers of graphene the energy gap strongly depends on the choice of stacking, and we found that the gap for the different types of stacking is much larger as compared to the case of Bernal stacking. Trigonal warping changes the size of the induced electronic gap by approximately $30 \%$ for intermediate and large values of the induced electron density.
\end{abstract}

DOI: 10.1103/PhysRevB.81.115432

PACS number(s): 73.22.Pr

\section{INTRODUCTION}

Graphene is a single layer of carbon atoms with hexagonal symmetry. ${ }^{1}$ Multilayers of graphene can be stacked differently depending on the horizontal shift between consecutive graphene planes, leading to very different electronic properties, ${ }^{2}$ e.g., to various band structures.

A perpendicular electric field applied to bilayer graphene, with the $A B$ stacking, can open an electronic gap between the valence and conduction bands. ${ }^{3}$ This was shown indirectly by transport measurements. ${ }^{4,5}$ Later on, spectroscopic measurements confirmed the opening of a gap in the energy spectrum. ${ }^{6-10}$ The extension of these bilayer results to three and four layers of graphene was presented in Ref. 11 in the case the perpendicular electric field was realized by a single gate. It was found that such an electric field causes an energy gap which was found to be a nonmonotonic function of the gate voltage, and a re-entrant opening and closing of the gap was predicted as a function of the electric field strength. In Ref. 12 the electronic band structure of the $A B A$-stacked trilayer graphene in the presence of back and top gates was investigated.

Recently, we generalized our previous results ${ }^{11}$ to the case when two, i.e., top and back, gates were applied to three as well as to four layers of graphene systems. ${ }^{13}$ We found that due to the trigonal warping the obtained results do not exhibit electron-hole symmetry. A nonmonotonic dependence of the true energy gap in trilayer graphene on the charge density on the gates was found. We also predicted an indirect gap with a nonmonotonic dependence on the gate voltage. Four layers of graphene exhibit a larger energy gap as compared to the three layer system, which is a consequence of the fact that Dirac fermions are present in the $A B$ stacked graphene multilayers in case of an odd number of layers, while for an even number of stacked graphene layers only charge carriers with a parabolic dispersion are present at low energies. ${ }^{14}$

Using Raman spectroscopy measurements the graphitic flake thickness, i.e., the number of graphene layers, can be obtained as was demonstrated in Refs. 15 and 16. In Ref. 15 a tunable three-layer graphene single-electron transistor was experimentally realized showing a transport gap near the charge neutrality point. Up to now, no four layer system was studied experimentally. Electrical tunable energy gap systems are of interest from a fundamental point of view but also for possible applications in electronics (e.g., for transistors) and photonics (i.e., wavelength tuning of a laser).

The electronic low-energy band structure of the $A B C$ stacked multilayer graphene was studied within an effective mass approximation in Ref. 17, with special attention to the Lifshitz transition, in which the Fermi circle breaks up into several pockets.

In this paper we study the effect of different ways of stacking of multilayers of graphene on the electric field induced band gap by top and back gates. We limit ourselves to those stackings that have been found in graphite. The Bernal stacking $(A B A)$, which has hexagonal symmetry, is common and stable, but some parts of graphite can also have rhombohedral one (the $A B C$ stacking). ${ }^{18}$ The band structure of three and four layer graphene systems in the presence of a perpendicular electric field is obtained using a tight-binding approach, where we used a self-consistent Hartree approximation to calculate the induced charges on the different graphene layers. We found that the gap for trilayer graphene with the $A B C$ stacking is much larger than the one for the $A B A$ stacking, which was studied in Ref. 13.

Similarly for four layers of graphene the energy gap also strongly depends on the choice of stacking and is smallest in case of Bernal stacking. When taking into account the circular asymmetry of the spectrum, which is a consequence of the trigonal warping, we found considerable changes in the size of the induced electronic gap for the considered systems at intermediate and high densities of electrons induced on the layers.

This paper is organized as follows. A short overview of our tight-binding approach with a description of the selfconsistent calculation are given in Sec. II for the $A B C$ stacked three layer graphene in the presence of top and back gates. The corresponding numerical results are also discussed here. In Sec. III we investigate four layer graphene with different stacking order in the presence of top and bottom gates. Section IV summarizes our conclusions. 

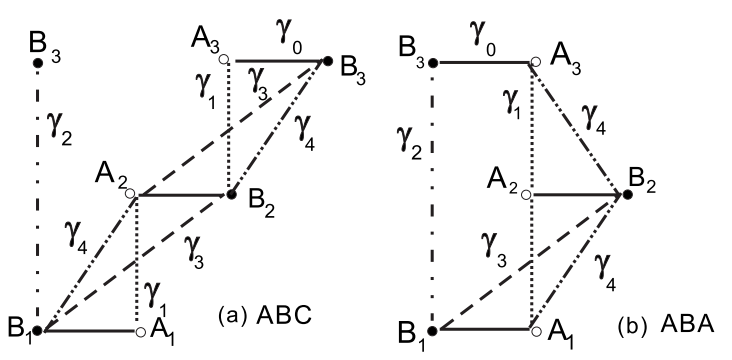

FIG. 1. Schematic of the different couplings between the sites for three layers of graphene, where $A$ sites are indicated by white circles and $B$ sites by black dots for: (a) the $A B C$ stacking and (b) the $A B A$ stacking.

\section{THREE LAYER GRAPHENE WITH THE $A B C$ STACKING IN AN EXTERNAL ELECTRIC FIELD}

We consider a system consisting of three layers of graphene with the $A B C$ stacking, which is modeled as three coupled hexagonal lattices with inequivalent sites $A_{i}$ and $B_{i}$ ( $i=1,2,3$ is the layer number) with $A_{1}$ and $A_{2}$, as well as $A_{3}$ and $B_{2}$ atoms on top of each other, as shown in Fig. 1. We use the Slonczewski-Weiss-McClure (SWMcC) parameters, i.e., $\gamma_{0}, \gamma_{1}, \gamma_{2}, \gamma_{3}, \gamma_{4}$ of tight-binding couplings for bulk graphite. Within each layer the interaction between nearestneighbor $A_{i}$ and $B_{i}$ atoms is described by the parameter $\gamma_{0}$. The strong coupling between nearest layers, i.e., between $A_{1}-A_{2}$ and $B_{2}-A_{3}$ atoms that lie directly above or below each other is given by $\gamma_{1}$ and the weaker nearest layer coupling by $\gamma_{3}\left(\gamma_{4}\right)$, i.e., between sites $B_{1}-B_{2}$ and $A_{2}-B_{3}\left(B_{1}\right.$ $-A_{2}, A_{1}-B_{2}, A_{2}-A_{3}$, and $\left.B_{2}-B_{3}\right)$. The interaction between the next nearest layers $\left(B_{1}-B_{3}\right)$ is determined by $\gamma_{2}$, as is shown in Fig. 1(a) and for comparison in Fig. 1(b) we show the unit cell for the $A B A$ trilayer. Using these parameters we compose the tight-binding Hamiltonian for three layer graphene with the $A B C$ stacking, which has the form ${ }^{19}$

$$
H=\left(\begin{array}{ccc}
D_{1} & H_{12} & H_{13} \\
H_{21} & D_{2} & H_{23} \\
H_{31} & H_{32} & D_{3}
\end{array}\right),
$$

where the rows and columns are ordered according to atom $A$ from layer 1, atom $B$ from layer 1, atom $A$ from layer 2, atom $B$ from layer 2, etc., with the following two by two matrixes:

$$
\begin{gathered}
D_{1}=\left(\begin{array}{cc}
0 & \gamma_{0} f \\
\gamma_{0} f^{*} & 0
\end{array}\right), \quad D_{2}=D_{1}^{\dagger}, \\
H_{12}=\left(\begin{array}{cc}
\gamma_{1} & -\gamma_{4} f^{*} \\
-\gamma_{4} f^{*} & \gamma_{3} f
\end{array}\right), \quad H_{21}=\left(\begin{array}{cc}
\gamma_{1} & -\gamma_{4} f \\
-\gamma_{4} f & \gamma_{3} f^{*}
\end{array}\right), \\
H_{32}=H_{23}^{\dagger}=\left(\begin{array}{cc}
-\gamma_{4} f & \gamma_{1} \\
\gamma_{3} f^{*} & -\gamma_{4} f
\end{array}\right), \quad H_{31}=H_{13}=\left(\begin{array}{cc}
0 & 0 \\
0 & \gamma_{2} / 2
\end{array}\right), \\
D_{3}=D_{2},
\end{gathered}
$$

$$
f\left(k_{x}, k_{y}\right)=e^{i k_{x} a_{0} / \sqrt{3}}+2 e^{-i k_{x} a_{0} / 2 \sqrt{3}} \cos k_{y} a_{0} / 2,
$$

with $a_{0}=2.46 \AA$ the in-plane lattice vector length. The Hamiltonian for the $A B A$ stacking was discussed in Ref. 13.

To control the density of electrons on the different graphene layers and independently the Fermi energy of the system, a top gate with a density of negative charges $n_{t}>0$ (the electron excess density is positive) on it, and a back gate with a density of positive charges $n_{b}<0$ are applied to the trilayer (a schematic picture was presented in Fig. 1 of Ref. 13). As a result a total excess density $n=n_{1}+n_{2}+n_{3}$ is induced $\left(n=n_{t}+n_{b}\right)$, with $n_{1}$ is the excess density on the closest layer to the top gate, $n_{3}$ on the closest layer to the back gate, and $n_{2}$ is the excess density on the middle layer. In our model the top or back gate produces a uniform electric field $E_{t, b}$ $=n_{t, b} e / 2 \varepsilon_{0} \kappa$, and due to the induced charges on the graphene layers, in its turn create fields $E_{i}=n_{i} e / 2 \varepsilon_{0} \kappa$, with $\varepsilon_{0}$ is the permittivity of vacuum and $\kappa$ is the dielectric constant. There is a simple relation between the charge density on the gates and the voltage between the gate and the closest graphene layer: $V_{t, b}=e n_{t, b} d / 2 \varepsilon_{0} \kappa$, where $d$ is the distance from the gate to the closest graphene layer (usually $d$ is equal to the oxide thickness, which is typically about $300 \mathrm{~nm}$ ). For our numerical calculations we use the value $\kappa=2.3$, which corresponds to graphene layers on $\mathrm{SiO}_{2}$. The difference between the charge densities induced on the individual layers of graphene creates asymmetries between the first and the second layers, as well as between the second and the third layers, which are determined by the corresponding change in the potential energies $\Delta_{1,2}$ and $\Delta_{2,3}$

$$
\begin{gathered}
\Delta_{1,2}(n)=\alpha\left(n_{2}+n_{3}-\left|n_{b}\right|\right), \\
\Delta_{2,3}(n)=\alpha\left(n_{3}-\left|n_{b}\right|\right),
\end{gathered}
$$

where $\alpha=e^{2} c_{0} / \varepsilon_{0} \kappa$, with $c_{0}=3.35 \AA$ is the inter-layer distance. The Hamiltonian Eq. (1) in the presence of the top and back gates is modified, and we have to add $\Delta_{1,2}(n)$ and $-\Delta_{2,3}(n)$ to the first and third layer on-site elements in Eq. (1). The tight-binding Hamiltonian operates in the space of coefficients of the tight-binding functions $c(\vec{k})$ $=\left(c_{A_{1}}, c_{B_{1}}, c_{A_{2}}, c_{B_{2}}, c_{A_{3}}, c_{B_{3}}\right)$, where $c_{A_{i}}=c_{A_{i}}(\vec{k})$ and $c_{B_{i}}$ $=c_{B_{i}}(\vec{k})$ are the $i$ th layer coefficients for $A$ and $B$ type of atoms, respectively. The total eigenfunction of the system is then given by

$$
\Psi_{\vec{k}}(\vec{r})=\sum_{i=1}^{N_{l}} c_{A_{i}} \psi_{\vec{k}}^{A_{i}}(\vec{r})+\sum_{i=1}^{N_{l}} c_{B_{i}} \psi_{\vec{k}}^{B_{i}}(\vec{r}),
$$

with $N_{l}$ is the number of layers. By diagonalizing the Hamiltonian one can obtain the six coefficients [in Eq. (5)] for fixed values of the layer asymmetries, from which we obtain the excess electronic densities on the individual layers:

$$
n_{i}=\frac{2}{\pi} \int d k_{x} d k_{y}\left(\left|c_{A_{i}}\right|^{2}+\left|c_{B_{i}}\right|^{2}\right) .
$$

The coefficients $c_{A_{i}}$ and $c_{B_{i}}$ depend on the energetic band index. Here we are interested in the case when the Fermi energy is located in the band gap, and in order to find the

where 


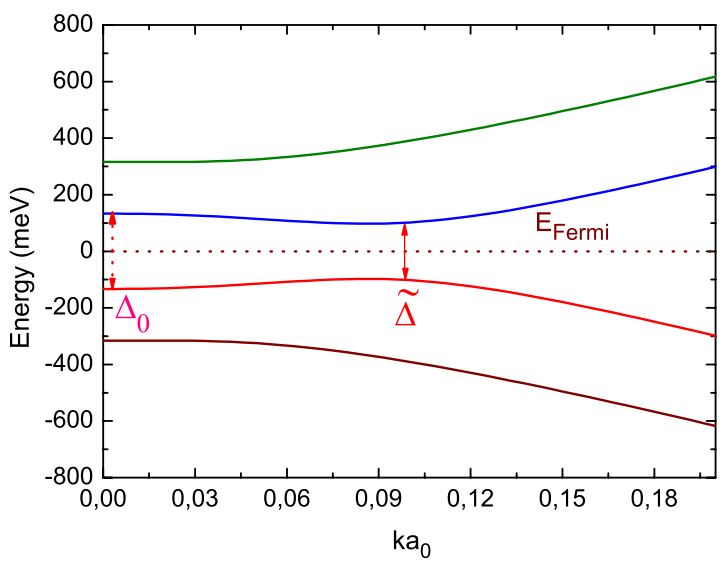

FIG. 2. (Color online) The circular symmetric band structure of trilayer graphene with the $A B C$ stacking order around the $K$-point when charges on the top and back gate are opposite but equal in magnitude, i.e., $-n_{b}=n_{t}=10^{13} \mathrm{~cm}^{-2}$, for the case when only $\gamma_{0}, \gamma_{1} \neq 0$. Horizontal dotted line is the Fermi level. The true gap $\widetilde{\Delta}$ and the energy gap at the $K$-point $\Delta_{0}$ are indicated.

redistribution of the electron density over the different layers in the valence bands one should integrate Eq. (6) over the Brillouin zone. The Fermi energy can be tuned into the opened gap when the magnitudes of the top and back gates are equal to each other but with opposite charges on them. The other case when the Fermi energy is located in the conduction or valence band was discussed in Ref. 13 for the $A B A$ stacked trilayer where we found that the obtained results do not exhibit electron-hole symmetry in the presence of trigonal warping. Using Eqs. (1)-(4) and (6) we evaluate the energy gap $\Delta_{0}$ at the $K$-point and the true gap, $\widetilde{\Delta}$, selfconsistently for a fixed total density $n_{t}+n_{b}=n_{1}+n_{2}+n_{3}$ (see Refs. 3 and 13).

In the following we will consider two cases. First, we neglect all interactions except between the nearestneighbor atoms in the same layer and between the atoms of adjacent layers which are on top of each other, i.e., we put $\gamma_{2}=\gamma_{3}=\gamma_{4}=\gamma_{5}=0$. This leads to a circular symmetric spectrum. In our calculations we used the parameter $\gamma_{0}$ $=3.12 \mathrm{eV}$ which leads to an in-plane velocity $v=\sqrt{3} \gamma_{0} a / 2 \hbar$ $\simeq 10^{6} \mathrm{~m} / \mathrm{s}$, and for the interlayer coupling strength, we take $\gamma_{1}=0.377 \mathrm{eV}$ (see Ref. 20). Second, the full interaction case is studied where the interaction between the different atoms is expressed by the SWMcC parameters $\left(\gamma_{2}=-0.0206, \gamma_{3}\right.$ $\left.=0.29, \gamma_{4}=0.12, \gamma_{5}=0.025\right)$, i.e., the effect of warping is included.

Figure 2 shows the band structure for trilayer graphene with the $A B C$ stacking when charges on the top and back gates are opposite but equal in magnitude with $-n_{b}=n_{t}$ $=10^{13} \mathrm{~cm}^{-2}$ when only $\gamma_{0}, \gamma_{1}$ are taken into account (with $\kappa=2.3$ ), and the Fermi energy is located in the forbidden gap. Notice that there is conduction band-valence band symmetry around the Fermi energy, and the true gap $\widetilde{\Delta}$ occurs away from the $K$-point where the gap is $\Delta_{0}=266 \mathrm{meV}>\widetilde{\Delta}$ $=195 \mathrm{meV}$. For the ABA stacking for the case when only $\gamma_{0}, \gamma_{1} \neq 0$ the true gap is zero for all densities.

When all the interactions between the different atoms are taken into account the surface of constant energy is no longer

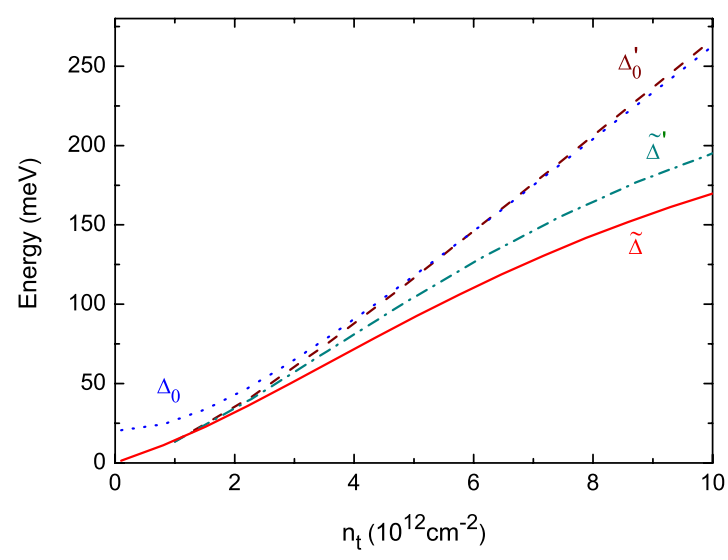

FIG. 3. (Color online) The dependence of the gap $\Delta_{0}$ (dotted blue curve) at the $K$-point, the true direct gap $\widetilde{\Delta}$ (solid red curve) for the $A B C$ trilayer graphene as a function of the top gate density $n_{t}$ providing the back gate density is $-n_{b}=n_{t}$. For comparison we show also the corresponding results, $\Delta_{0}^{\prime}$ (dashed red curve) and $\widetilde{\Delta^{\prime}}$ (dotdashed blue curve) when only $\gamma_{0}, \gamma_{1} \neq 0$.

circular. In Fig. 3 we show the gap $\Delta_{0}$ at the $K$-point (dotted blue curve) and the true direct gap $\widetilde{\Delta}$ (solid red curve) for trilayer graphene with the full interaction, as a function of the top gate density $n_{t}$ providing the back gate density $-n_{b}$ $=n_{t}$. For comparison in the same figure we show also the corresponding results, $\Delta_{0}^{\prime}$ (dashed red curve) and $\widetilde{\Delta^{\prime}}$ (dotdashed blue curve) when only $\gamma_{0}, \gamma_{1} \neq 0$. Notice, that for high densities $\left(-n_{b}=n_{t} \approx 10^{13} \mathrm{~cm}^{-2}\right)$ the inclusion of the full interaction leads to a lowering of the true gap by $30 \%$. It is interesting to note that similar values for the energy gaps and the relative difference between them were found for the case of bilayer $A B$ graphene: ${ }^{13}$ the true gap for the $A B$ bilayer at $-n_{b}=n_{t} \approx 10^{13} \mathrm{~cm}^{-2}$ is $142 \mathrm{meV}$ when $\kappa=2.3$ and $198 \mathrm{meV}$ for the case of $\kappa=1$ when the full interaction is included. These results compare with $169 \mathrm{meV}(\kappa=2.3)$ and $207 \mathrm{meV}$ $(\kappa=1)$ for our $A B C$ trilayer.

This similarity becomes more remarkable if we compare the layer densities induced by external gates for the $A B A$ and $A B C$ trilayers with the $A B$ bilayer. For the $A B A$ trilayer, when only a back gate was applied to the first layer, ${ }^{11}$ we found that $n_{1}=6.1, n_{2}=3.2$ and $n_{3}=1.2$ at $n_{b}=10$ (in units $10^{12} \mathrm{~cm}^{-2}$ ). The small amount of excess charges on the last layers was explained by the fact that the graphene layers screen the electric field and the layer asymmetries between the last layers, counted from the gate, are very small. The true gap for this system $(\widetilde{\Delta}=17 \mathrm{meV})$ is smaller in comparison with the bilayer case, where for the latter $n_{1}=6.7$ and $n_{2}=3.3(\widetilde{\Delta}=82 \mathrm{meV})$. Now, when only a back gate is applied to the $A B C$ trilayer we find that the densities on the second and the third layers (counted from the back gate) are very close to each other: $n_{2}=n_{3} \simeq 2$ at $n_{b}=10$ and $n_{1}=6.24$, which makes the $A B C$ system distribution and the gap (with $\widetilde{\Delta}=117 \mathrm{meV}$ ) similar to the $A B$ bilayer ones. In Fig. 1(a) one can see that in the case of the $A B C$ stacking there are never three atoms stacked on top of each other, as in the case for the $A B A$. As a result the electric field (of the gate located near the first graphene layer for the $A B C$ stacking) penetrates 


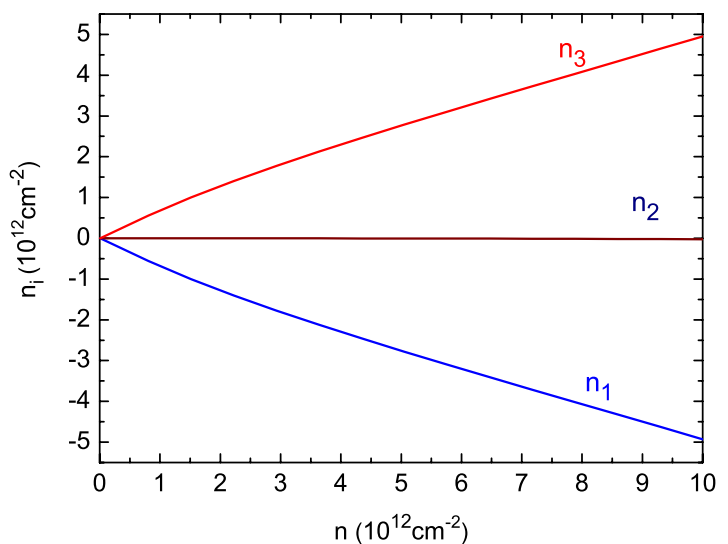

FIG. 4. (Color online) The charge density $n_{i}$ on the different graphene layers for the $A B C$ trilayer with $\kappa=2.3$ and with the full interaction included as a function of the charge density on the top gate $n_{t}$ with the back gate density $n_{b}=-n_{t}$.

easier to the last layers inducing excess charges, while for the $A B A$ stacking the electric field is much more strongly screened.

When both gates are applied to the $A B C$ trilayer graphene (when the full interaction is included) the excess charge densities at $-n_{b}=n_{t}=10$, shown in Fig. 4, on the outer layers are $-n_{1}=n_{3}=4.9$ and in the middle layer is zero. Notice that the excess charge densities on the bottom and the top layers are symmetric as in the case of the $A B$ bilayer, as well as the gaps have also similar values. While for the $A B A$ trilayer it was $n_{1}=-3.84$ and $n_{3}=3.67,{ }^{13}$ and $n_{2}=0.17$ when $-n_{b}=n_{t}$ $=10$; the inclusion of the full interaction in the $A B A$ case makes the excess electron density in the middle layer different from zero, and it opens a small gap about $5 \mathrm{meV}$. So, we see that the $A B C$ system has a large gap, comparable with the $A B$ bilayer one and behaves as a bilayer with shifted sheets, while the $A B A$ opens up much smaller gap and is similar to the case of an $A A$ stacked bilayer.

In the case of the previous studied $A B A$ trilayer ${ }^{11}$ the inclusion of trigonal warping leads to a nonmonotonic behavior of the gaps as a function of gate voltage, as well as a much stronger lowering of the true gap. Here, we found that the energy gaps for the $A B C$ stacked trilayer is much larger as compared to the case of the $A B A$ trilayer. Figure 5 shows a three-dimensional (3D)-plot and the corresponding contour plot of the highest valence band for three layer $A B C$ stacked graphene near the $K$-point ( $K$-point is chosen as the origin, $\kappa=2.3)$ for $n_{t}=-n_{b}=10^{13} \mathrm{~cm}^{-2}$. The lowest conduction band is again symmetric with the highest valence band just as in the case when only $\gamma_{0}, \gamma_{1} \neq 0$. Here, for the $A B C$ stacking we find three maxima but did not find additional maxima as in the case of the $A B A$ stacking $^{13}$ and as a result we do not observe an indirect gap.

\section{FOUR LAYER GRAPHENE SYSTEM IN AN EXTERNAL ELECTRIC FIELD}

Now, we consider the four layer graphene system, which can be arranged in many different ways as schematically shown in Figs. 6(a)-6(c). The tight-binding parameters $\gamma_{i}$

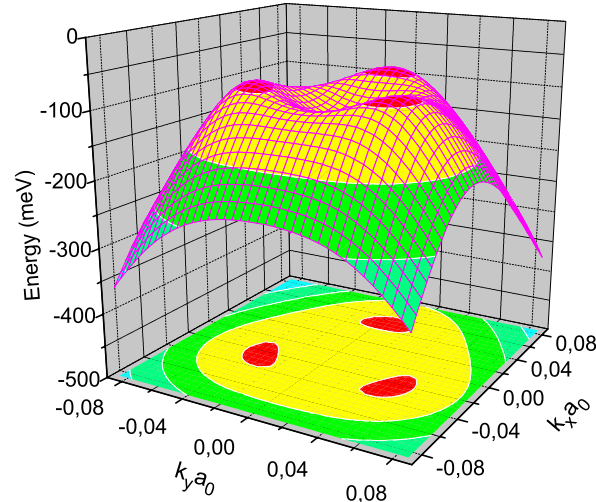

FIG. 5. (Color online) The highest valence band, with the corresponding contour plots for the $A B C$ stacked trilayer graphene near the $K$-point ( $K$-point is chosen as the origin) with equal but opposite charges on the top and back gate when $n_{t}=-n_{b}=10^{13} \mathrm{~cm}^{-2}$. The Fermi energy is located in the energy gap at $E=0$.

and the interaction between the individual carbon atoms for all these cases are indicated in these figures. Four layer graphene is described by the Hamiltonian

$$
H=\left(\begin{array}{cccc}
D_{1} & H_{12} & H_{13} & H_{14} \\
H_{21} & D_{2} & H_{23} & H_{24} \\
H_{31} & H_{32} & D_{3} & H_{34} \\
H_{41} & H_{42} & H_{43} & D_{4}
\end{array}\right),
$$

where $H_{i j}$ and $D_{i}$ with $i=1,2,3$ are the matrix elements of the $A B C$ trilayer given by Eqs. (2a) and (2b) and for the $A B C A$ stacking we have

$$
\begin{gathered}
H_{14}=H_{41}=\left(\begin{array}{ll}
0 & 0 \\
0 & 0
\end{array}\right), \quad H_{42}=H_{24}^{\dagger}=\left(\begin{array}{cc}
0 & 0 \\
\gamma_{2} / 2 & 0
\end{array}\right), \\
H_{43}=H_{34}^{\dagger}=H_{32}, \quad D_{4}=D_{3},
\end{gathered}
$$

while for the $A B C C$ stacking these matrixes have the following form:

$$
H_{14}=H_{41}=\left(\begin{array}{ll}
0 & 0 \\
0 & 0
\end{array}\right), \quad H_{42}=H_{24}^{\dagger}=\left(\begin{array}{cc}
0 & \gamma_{5} / 2 \\
0 & 0
\end{array}\right),
$$

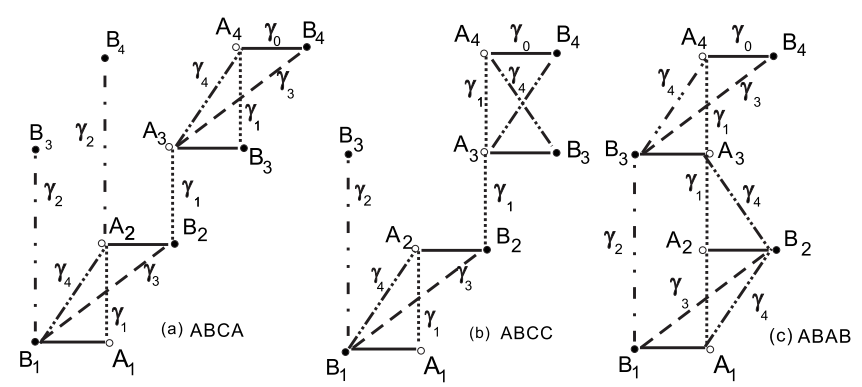

FIG. 6. Schematic of the couplings between the different ( $A$-white and $B$-black dots) sites for four layers of graphene for: (a) the $A B C A$, (b) the $A B C C$ stacking, and (c) the $A B A B$ Bernal stacking. 

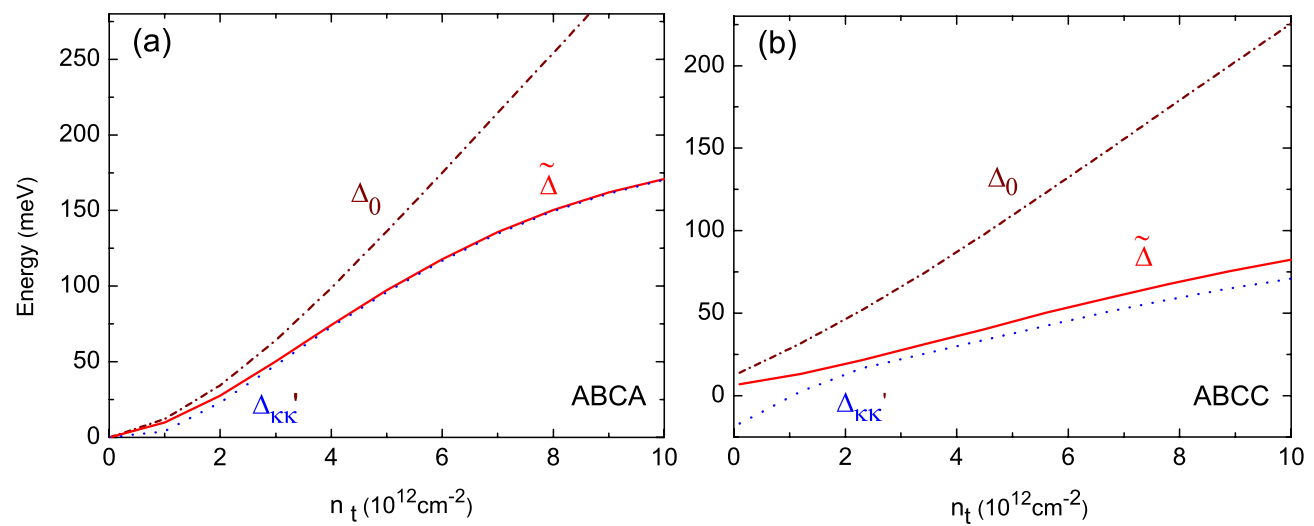

FIG. 7. (Color online) The dependence of the gap $\Delta_{0}$ at the $K$-point (dot-dashed red curve), the true direct gap $\widetilde{\Delta}$ (solid red curve), and the true indirect gap (dotted blue curve) $\Delta_{k k^{\prime}}$ as a function of the top gate density $n_{t}$ for four layer graphene where we included the full interaction. The back gate density $-n_{b}=n_{t}$ is the same (but opposite in sign) as the top gate. Results are shown for: (a) the $A B C A$ stacking and (b) the $A B C C$ stacking.

$$
H_{43}=H_{34}^{\dagger}=\left(\begin{array}{cc}
\gamma_{1} & -\gamma_{4} f^{*} \\
-\gamma_{4} f & \gamma_{1}
\end{array}\right), \quad D_{4}=D_{3} .
$$

We consider a four layer graphene system with top and back gates, which induce a total excess density $n=n_{1}+n_{2}+n_{3}+n_{4}$, where $n_{i}$ is the excess density on the $i$ th layer as counted from the top gate. The corresponding change in the potential energy between consecutive layers is

$$
\begin{gathered}
\Delta_{1,2}(n)=\alpha\left(n_{2}+n_{3}+n_{4}-\left|n_{b}\right|\right), \\
\Delta_{2,3}(n)=\alpha\left(n_{3}+n_{4}-\left|n_{b}\right|\right), \\
\Delta_{3,4}(n)=\alpha\left(n_{4}-\left|n_{b}\right|\right) .
\end{gathered}
$$

By adding $\Delta^{\mathrm{II}}=\Delta_{1,2}(n), \quad \Delta^{\mathrm{III}}=\Delta_{1,2}(n)+\Delta_{2,3}(n), \quad$ and $\Delta^{\mathrm{IV}}$ $=\Delta_{1,2}(n)+\Delta_{2,3}(n)+\Delta_{3,4}(n)$ to the on-site elements of the II, III, and IV layer of the $A B C A$ or the $A B C C$ four layer Hamiltonian, respectively, we obtain the Hamiltonian in the presence of top and bottom gates. The eight coefficients $c_{A_{i}}$ $=c_{A_{i}}(\vec{k})$ and $c_{B_{i}}=c_{B_{i}}(\vec{k})$ for fixed values of the layer asymmetries defined by Eqs. (10a)-(10c) can be obtained by diagonalizing the corresponding Hamiltonian. The electronic den- sities on the individual layers are given by Eq. (6). The gaps $\Delta_{0}$ and $\widetilde{\Delta}$ are evaluated self-consistently analogously as was done for the three layer system.

The variation in the gap $\Delta_{0}$ at the $K$-point (dot-dashed red curve), the true direct gap $\widetilde{\Delta}$ (solid red curve), and the true indirect gap (dotted blue curve) $\Delta_{k k^{\prime}}$ with the top gate density $n_{t}\left(n_{b}=-n_{t}\right)$ for four layer graphene with the full interaction is shown in Fig. 7(a) for the $A B C A$ stacked four layer graphene and in Fig. 7(b) for the $A B C C$ stacking. One can see that for the $A B C A$ stacking with full interaction the true direct gap is very close to the corresponding gap in the case of a trilayer with the $A B C$ stacking, e.g., for $n_{t}=-n_{b}=10^{13} \mathrm{~cm}^{-2}$ the true gap is about $171 \mathrm{meV}$ for four layer graphene with the $A B C A$ stacking and for the $A B C$ trilayer it is $169 \mathrm{meV}$. In Figs. 8(a) and 8(b) we present the layer densities for the $A B C A$ and $A B C C$ four layer graphene systems, respectively, and we include the curves for the densities in the $A B C$ stacked trilayer for comparison in both figures (dashed curves). It is remarkable that the excess densities for the $A B C A$ system on the outer as well as on the inner layers are symmetric. Notice that the densities, shown in Fig. 8(a), on the outer layers for the $A B C A$ are very close to the $A B C$ trilayer graphene ones
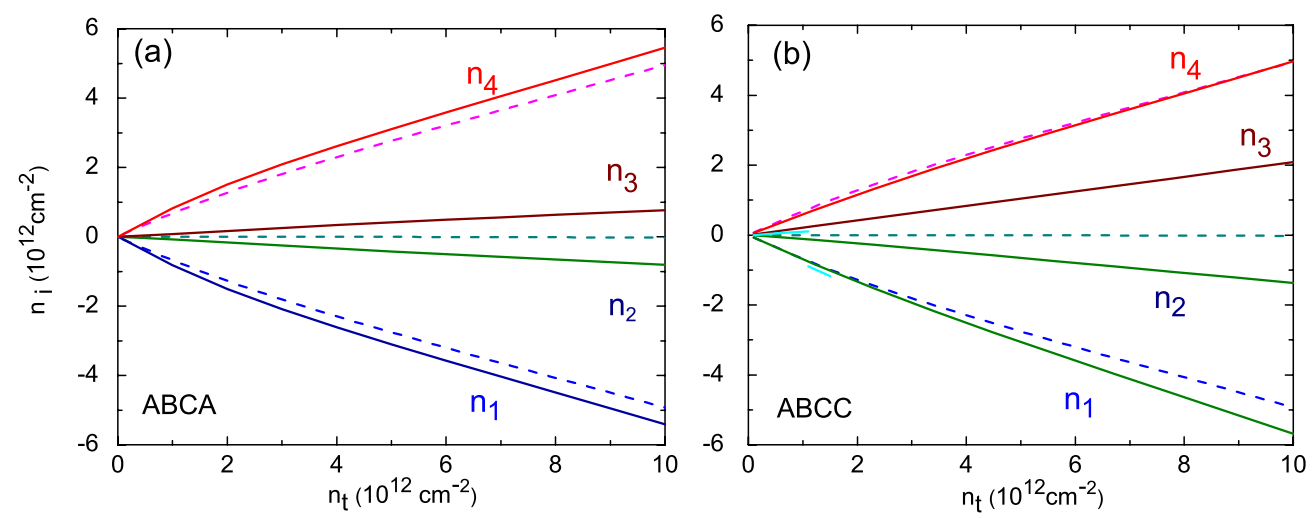

FIG. 8. (Color online) The layer densities $n_{i}$ (solid curves) for the four layer system as a function of the charge density on the top gate $n_{t}$ (providing $-n_{b}=n_{t}$ ) when the full interaction is included: (a) for the $A B C A$ stacking and (b) for the $A B C C$ stacking. In both cases we added the results for the layer densities $n_{i}^{\prime}$ (dashed curves) for the $A B C$ stacked trilayer when the full interaction is included. 
for all the values of $n_{t}$ : at $-n_{b}=n_{t}=10$ for the $A B C A$ $n_{4}=-n_{1}=5.4$, while for the $A B C$ trilayer graphene $n_{3}=-n_{1}$ $=4.9$ (in the units $10^{12} \mathrm{~cm}^{-2}$ ). We see that also the $A B C A$ four layer graphene behaves as the $A B$ bilayer. The localization of the atoms (see Fig. 6) can explain why the excess electron densities on the outer sheets for the $A B C A$ system are larger than the densities for the $A B C$ trilayer and even in comparison with the $A B$ bilayer densities (which has $n_{2}=$ $-n_{1}=3.7$ at $-n_{b}=n_{t}=10$ ). For the $A B C A$ system there are never four atoms on top of each other, as is the case for the $A B A B$ stacking. As a consequence, the electric field of the top gate, e.g., at $A_{3}$ [see Fig. 6(a)] is screened only by the $B_{2}$ atom. Similarly, the field of the back gate at $B_{2}$ is screened only by $A_{3}$ atom. As a result, both these atoms feel the field of the top as well as the back gate, which leads to a decrease in excess charges on the inner layers (i.e., to a neutralization of these charges by the opposite gates). However, an outer layer (of the $A B C A$ system) which is mainly charged by its closest gate does not feel the further located gate since the latter is screened by the inner layers. In the $A B$ bilayer the two sheets feel both gates, and consequently the excess charges (by absolute value) are less than in the outer layers of the $A B C A$. Due to this, also the gap for the $A B$ bilayer is less (see the gap value in previous section) than the $A B C A$ one (for the same strength of the top and back gates). We see also, that the gap is large when the amount of excess charges in the inner layers is small (as it is for the $A B C A$ system). We found also that for $\kappa=1$ the true gap is $183 \mathrm{meV}$ for the $A B C A$; the relative difference with the case of $\kappa=2.3$ is only about $5 \%$.

In contrast, for the $A B C C$ four layer graphene the excess density on the third layer is larger, and on the fourth layer is smaller than the corresponding densities found in the case of the $A B C A$ system. So, the increase in the excess densities as well as the density asymmetry in the inner sheets leads to a decrease in the gap. Also, the fact that the third and fourth sheets are not shifted, i.e., they have the $A A$ stacking order, explain that in the $A B C C$ four layer graphene the electric field opens up a smaller gap. In both cases we found a much larger gap (about $170 \mathrm{meV}$ for the $A B C A$ stacking and 70 meV for the $A B C C$ stacking at $n_{t}=-n_{b}=10^{13} \mathrm{~cm}^{-2}$ ) than in the case of the $A B A B$ stacked four layer graphene ${ }^{13}$ (with 5 $\mathrm{meV}$ for the same density). So, we see that from all the systems, considered in this paper and in Ref. 13, the Bernal stacking leads to the smallest gap.

Figures 9(a) and 9(b) show 3D plots and corresponding contour plots of the highest valence and the lowest conduction bands near the $K$-point ( $K$-point is chosen as the origin, $\kappa=2.3)$ in the case of $n_{t}=-n_{b}=10^{13} \mathrm{~cm}^{-2}$, for the $A B C A$ and the $A B C C$ stacking, respectively. The conduction band for the $A B C C$ stacking has a "Mexican hat" shape maxima and minima on a ring, as shown in the contour plot, e.g., there is a minimum at $k_{x} a_{0} \simeq-0.17$ and $k_{y} a_{0}=0$. In its turn the valence band has a local minimum between the two maxima at the plane $k_{x} a_{0} \simeq-0.17$. The asymmetry between the contour plots for the conduction and the valence bands for the $A B C C$ [see Fig. 9(b)] leads to an indirect true gap. At low densities there is a true direct gap for the $A B C C$ but due to the overlap
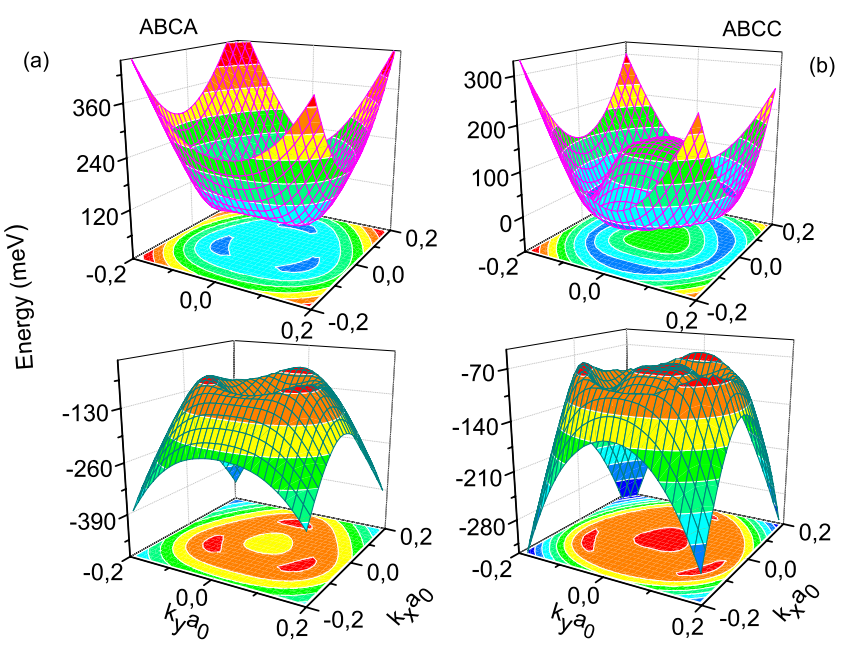

FIG. 9. (Color online) 3D plots and corresponding contour plots of the highest valence band (bottom figures) and the lowest conduction band (top figures) around the $K$-point ( $K$-point is chosen as the origin $\kappa=2.3$ ) when $n_{t}=-n_{b}=10^{13} \mathrm{~cm}^{-2}$ for: (a) the $A B C A$ and (b) the $A B C C$ stacking of four layers of graphene.

between the bands at different points in $k$ space the indirect gap is negative as is shown in Fig. 7(b), i.e., we have a semimetal for low gate densities. For the $A B C A$ we find only three minima in the conduction band and a symmetric valence band [see Fig. 9(a)], analogously with the $A B C$ trilayer case. For the $A B C A$ system the indirect gap is smaller than the direct one at low densities, and they coincide at high densities.

When finishing this paper we came aware of a recent preprint ${ }^{21}$ on the effect of an electric field on multilayers of graphene with different stacking. They used the simplest approximation where only $\gamma_{0}, \gamma_{1} \neq 0$. They argued that the inclusion of the other tight-binding parameters do not affect strongly the band structure and the true gap. However, our calculations show that the true gap can be changed by $30 \%$.

\section{CONCLUSIONS}

The effect of different stacking order on the electric field induced energy gap of three and four layers of graphene was investigated. For three-as well as for four-layer graphene the energy gap strongly depends on the choice of stacking, and we found that the gap is much larger than for the previously studied Bernal stacking. We found that the true gap for the $A B C$ trilayer and the $A B C A$ four layer graphene is comparable with the corresponding gap for bilayer graphene with Bernal stacking. The account of the circular asymmetry of the spectrum, which is a consequence of the trigonal warping, considerably changes the size of the induced electronic gap for the studied systems.

This work was supported by the Flemish Science Foundation (FWO-Vl), the "Belgian Science Policy" IAPprogram, and the Brazilian Science Foundation CNPq. One of us (A.A.A.) was supported by the Belgian Federal Science Policy Office. 
*artak.avetisyan@ua.ac.be

†bart.partoens@ua.ac.be

¥francois.peeters@ua.ac.be

${ }^{1}$ K. S. Novoselov, A. K. Geim, S. V. Morozov, D. Jiang, M. I. Katsnelson, I. V. Grigorieva, S. V. Dubonos, and A. A. Firsov, Nature (London) 438, 197 (2005).

${ }^{2}$ S. Latil and L. Henrard, Phys. Rev. Lett. 97, 036803 (2006).

${ }^{3}$ E. McCann, Phys. Rev. B 74, 161403(R) (2006).

${ }^{4}$ E. V. Castro, K. S. Novoselov, S. V. Morozov, N. M. R. Peres, J. M. B. Lopes dos Santos, J. Nilsson, F. Guinea, A. K. Geim, and A. H. Castro Neto, Phys. Rev. Lett. 99, 216802 (2007).

${ }^{5}$ J. B. Oostinga, H. B. Heersche, X. Liu, A. F. Morpurgo, and L. M. K. Vandersypen, Nature Mater. 7, 151 (2008).

${ }^{6}$ T. Ohta, A. Bostwick, T. Seyller, K. Horn, and E. Rotenberg, Science 313, 951 (2006).

${ }^{7}$ Z. Q. Li, E. A. Henriksen, Z. Jiang, Z. Hao, M. C. Martin, P. Kim, H. L. Stormer, and D. N. Basov, Phys. Rev. Lett. 102, 037403 (2009).

${ }^{8}$ L. M. Zhang, Z. Q. Li, D. N. Basov, M. M. Fogler, Z. Hao, and M. C. Martin, Phys. Rev. B 78, 235408 (2008).

${ }^{9}$ A. B. Kuzmenko, E. van Heumen, D. van der Marel, P. Lerch, P. Blake, K. S. Novoselov, and A. K. Geim, Phys. Rev. B 79,
115441 (2009).

${ }^{10}$ Y. Zhang, T.-T. Tang, C. Girit, Z. Hao, M. C. Martin, A. Zettl, M. F. Crommie, Y. R. Shen, and F. Wang, Nature (London) 459, 820 (2009).

${ }^{11}$ A. A. Avetisyan, B. Partoens, and F. M. Peeters, Phys. Rev. B 79, 035421 (2009).

${ }^{12}$ M. Koshino and E. McCann, Phys. Rev. B 79, 125443 (2009).

${ }^{13}$ A. A. Avetisyan, B. Partoens, and F. M. Peeters, Phys. Rev. B 80, 195401 (2009).

${ }^{14}$ B. Partoens and F. M. Peeters, Phys. Rev. B 75, 193402 (2007).

${ }^{15}$ J. Guttinger, C. Stampfer, F. Molitor, D. Graf, T. Ihn, and K. Ensslin, New J. Phys. 10, 125029 (2008).

${ }^{16}$ D. Graf, F. Molitor, K. Ensslin, C. Stampfer, A. Jungen, C. Hierold, and L. Wirtz, Nano Lett. 7, 238 (2007).

${ }^{17}$ M. Koshino and E. McCann, Phys. Rev. B 80, 165409 (2009).

${ }^{18}$ H. Lipson and A. R. Stokes, Proc. R. Soc. London, Ser. A 181, 101 (1942).

${ }^{19}$ F. Guinea, A. H. Castro Neto, and N. M. R. Peres, Phys. Rev. B 73, 245426 (2006).

${ }^{20}$ B. Partoens and F. M. Peeters, Phys. Rev. B 74, 075404 (2006).

${ }^{21}$ M. Koshino, arXiv:0911.3484 (unpublished). 